\title{
Selenium Status of the Australian Population: Effect of Age, Gender and Cardiovascular Disease
}

\author{
Robyn Lymbury • Ujang Tinngi • Lyn Griffiths • \\ Franklin Rosenfeldt • Anthony V. Perkins
}

Abstract Selenium (Se) is an essential trace element and the clinical consequences of Se deficiency have been well-documented. Se is primarily obtained through the diet and recent studies have suggested that the level of Se in Australian foods is declining. Currently there is limited data on the Se status of the Australian population so the aim of this study was to determine the plasma concentration of Se and glutathione peroxidase (GSH-Px), a wellestablished biomarker of Se status. Furthermore, the effect of gender, age and presence of cardiovascular disease (CVD) was also examined. Blood plasma samples from healthy subjects ( 140 samples, mean age $=54$ years; range, $20-86$ years $)$ and CVD patients $(112$ samples, mean age $=67$ years; range, $40-87$ years) were analysed for Se concentration and GSH-Px activity. The results revealed that the healthy Australian cohort had a mean plasma Se level of $100.2 \pm$ $1.3 \mu \mathrm{g} \mathrm{Se} / \mathrm{L}$ and a mean GSH-Px activity of $108.8 \pm 1.7 \mathrm{U} / \mathrm{L}$. Although the mean value for plasma Se reached the level required for optimal GSH-Px activity (i.e. $100 \mu \mathrm{g} \mathrm{Se} / \mathrm{L}$ ), $47 \%$ of the healthy individuals tested fell below this level. Further evaluation revealed that certain age groups were more at risk of a lowered Se status, in particular, the oldest age group of over 81 years $($ females $=97.6 \pm 6.1 \mu \mathrm{g} \mathrm{Se} / \mathrm{L}$; males $=89.4 \pm 3.8 \mu \mathrm{g} \mathrm{Se} / \mathrm{L}$ ). The difference in Se status between males and females was not found to be significant. The presence of CVD did not appear to influence Se status, with the exception of the over 81 age group, which showed a trend for a further decline in Se status with disease (plasma Se, 93.5 $\pm 3.6 \mu \mathrm{g} \mathrm{Se} / \mathrm{L}$ for healthy versus $88.2 \pm 5.3 \mu \mathrm{g} \mathrm{Se} / \mathrm{L}$ for CVD; plasma GSH-Px, $98.3 \pm 3.9 \mathrm{U} / \mathrm{L}$ for healthy versus $87.0 \pm$ 6.5 U/L for CVD). These findings emphasise the importance of an adequate dietary intake of Se for the maintenance of a healthy ageing population, especially in terms of cardiovascular health.

R. Lymbury $\cdot$ L. Griffiths $\cdot$ A. V. Perkins $(\bowtie)$

Heart Foundation Research Centre, School of Medical Science, Griffith University Gold Coast Campus, Parklands Drive, Southport, QLD 9726, Australia e-mail: A.Perkins@griffith.edu.au

Q1 U. Tinngi

Centre for Public Health Sciences, Queensland Health Forensic and Scientific Services,

39 Kessels Road, Coopers Plains, QLD 4108, Australia 
Keywords Selenium $\cdot$ Glutathione peroxidase $\cdot$ Elderly $\cdot$ Cardiovascular disease $\cdot$ Australia

\section{Introduction}

The importance of maintaining a healthy Se status has been well-established, with Se deficiency diseases documented in both animals and humans. Furthermore, moderate to severe Se deficiency has been associated with increased incidence of several diseases, including certain cancers [1-4] and cardiovascular diseases (CVD) [5-7]. These diseases are responsible for the majority of deaths in developed countries with age being a common risk factor. Se levels have been shown to decline with age, even in healthy populations, and a lowered Se status has been linked with increased overall mortality [4, 8]. Currently the recommended daily intakes for selenium in Australia are $85 \mu \mathrm{g} /$ day for males and $75 \mu \mathrm{g} /$ day for females.

Food is the major source of Se and food Se concentrations are directly influenced by the level of Se in the soil from which the food was derived. Globally, soil Se levels are highly variable [8] and this is also the case within Australia (Fig. 1) [9]. Regions in central Queensland and Cape York Peninsula have been identified as seleniferous, whilst substantial food producing regions of south east Queensland have been identified as Se deficient. Other regions in Australia that have been identified as Se poor include the Southern, Central and Northern Tablelands of NSW, the south-east coast of Queensland, south-west Western Australia, coastal and central regions of Victoria, most of Tasmania, as well as areas of South Australia, including Kangaroo Island and Mount Lofty Ranges [9, 10]. Large areas of Australia have not yet been mapped for soil Se and it is possible that the areas at risk of Se deficiency are much greater [10].

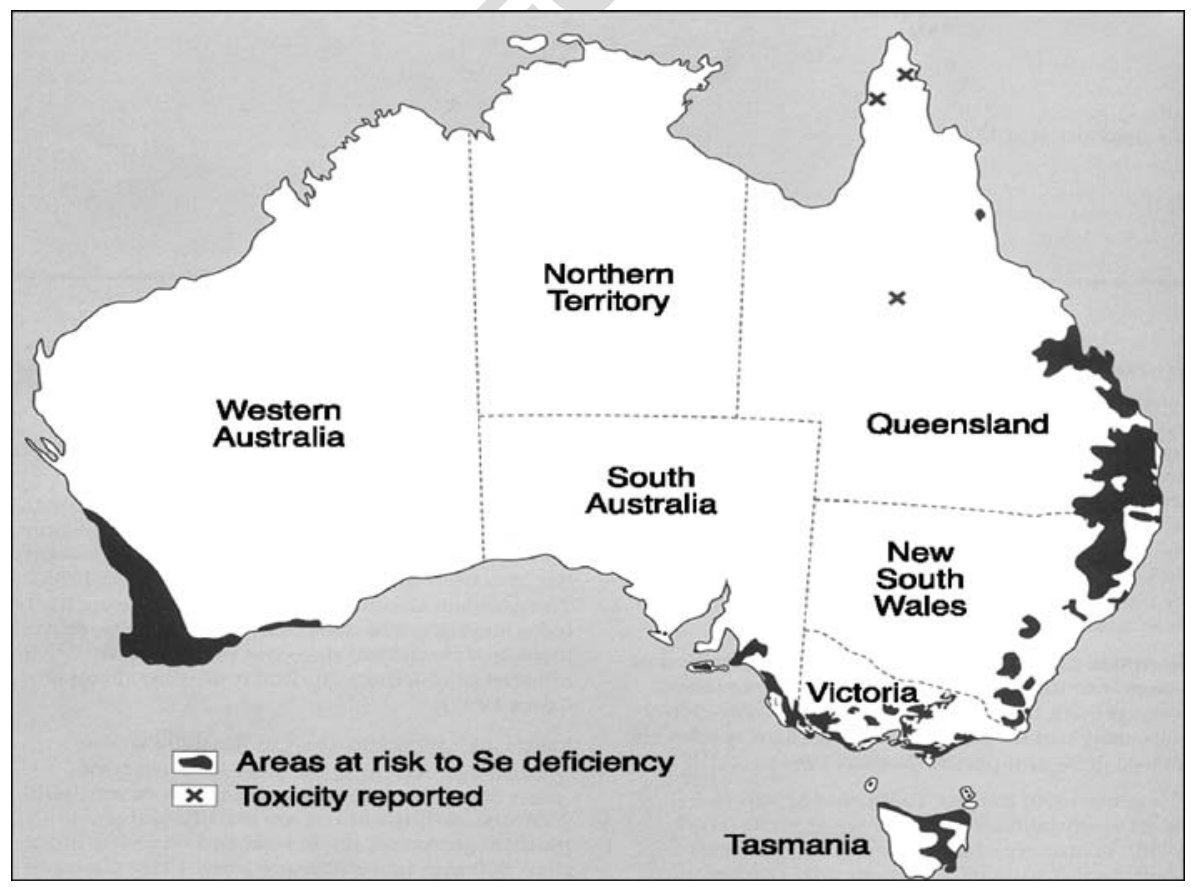

Fig. 1 Se in Australian soils (from [9]) 
Selenium Status of the Australian Population

There have been several studies on the Se status of the Australian population. The mean 55 plasma estimate derived from these studies is $94 \mu \mathrm{g} / \mathrm{L}$, however post 1990 data has indicated a mean plasma level of $89 \mu \mathrm{g} / \mathrm{L}$. Given the recent evidence demonstrating declining Se content in the Australian food supply it is important that the Se status be monitored every 5-10 years. Furthermore many of the previously published studies examined a local population and not populations from across the country. In this study we have measured levels of plasma Se as well as the activity of the selenoprotein glutathione peroxidase (GSH-Px) [11,12] in two cohorts of Australians and examined the influence of age, sex and the presence of CVD on Se status.

\section{Materials and Methods}

This was a simple, cross-sectional study on cardiac surgery patients and healthy controls, matched for age and gender. Blood plasma samples were collected from healthy volunteers. In all 140 plasma samples were collected, with 20 samples per age group $(n=10$ males and 10 females per group). The age groups were as follows: $20-30$ years, $31-40$ years, $41-50$ years, 51-60 years, $61-70$ years, $71-80$ years, and 81 years and over. Blood plasma samples were also collected from patients presenting with cardiovascular disease. There were 112 plasma samples collected in total and the sample numbers per age group were as follows: 41-50 years $(n=11)$, 51-60 years $(n=29), 61-70$ years $(n=35), 71-80$ years $(n=28)$, and 81 years and over $(n=9)$. Patients were excluded if they had used antioxidants within 1 month of sample collection.

\section{Se Determination}

The levels of selenium in blood plasma were analysed by inductively coupled plasma mass spectrometry (ICP-MS), as described in Navarro_Alarcon and Lopez_Martinez [2]. The high lipid and protein levels in blood plasma were initially broken down by the addition of Triton $\mathrm{X}-100(0.01 \%)$, to prevent spectral and matrix interferences during the ICP-MS analysis. The level of selenium in blood was analysed as Se-82 isotope, and for improved sensitivity and stability, an internal standard of germanium was used. The certified reference materials of Whole Blood Seronorm Trace Elements (SERO, Norway) and UTAK (USA) were used for quality control for the recovery of selenium.

Glutathione peroxidase activity was determined by a modified method of Paglia and Valentine (1967). Activity was determined spectrophotometrically by coupling the oxidation of glutathione and nicotinamide adenine dinucleotide phosphate (NADPH) using glutathione reductase. Briefly, $900 \mu \mathrm{l}$ of assay mix containing $0.1 \mathrm{M}$ potassium phosphate $(\mathrm{KPi}) \mathrm{pH} 7.0$, $2 \mathrm{mM}$ ethylenediaminetetraacetic acid (EDTA), $0.5 \mathrm{U} / \mathrm{ml}$ glutathione reductase, $10 \mathrm{mM}$ glutathione and $0.3 \mathrm{mM}$ reduced NADPH was placed into a Varian CARY 50 spectrophotometer set at $340 \mathrm{~nm}$. Diluted tissue extracts ( $50 \mu \mathrm{l}$ of $1 / 10$ dilution) was added to the cuvette along with $50 \mu \mathrm{l}$ of $20 \mathrm{mM}$ tert-butyl hydroperoxide a suitable substrate for glutathione peroxidase. The decrease in A340 was determined over a 3-min period and rate calculations were performed using Varian Cary software. Glutathione peroxidase activity was standardised against protein concentrations and expressed as mmoles per minute per mg of protein (mmoles/min/mg). 
All data were analysed using either unpaired two-tailed Student $t$-test or one-way ANOVA as described in Chapter 2, section 2.7. Values represent mean \pm SEM unless otherwise indicated.

\section{Results}

Se Status of Healthy Australians

The mean plasma Se concentration for the healthy population 20 to 86 years (mean age= 54 years) was found to be $100.2 \pm 1.3 \mu \mathrm{g} \mathrm{Se} / \mathrm{L}$. For the same population, the mean GSH-Px activity of plasma was found to be $108.8 \pm 1.7 \mathrm{U} / \mathrm{L}$. A plasma Se level of $100 \mu \mathrm{g} / \mathrm{L}$ plasma is required for maximal plasma GSH-Px activity $[8,11,13]$ and analysis of the study data revealed that $53 \%$ of healthy Australians tested had Se levels above this value. There was no significant difference detected between males and females for either plasma Se $(p=0.26)$ or GSH-Px $(p=0.33)$, however the values for the males were slightly lower than those found for females.

Se status did vary with age, as shown in Fig. 2. The overall trend suggests a bell-curve effect, with lower Se levels seen in the youngest (20-30 years) and oldest (over 81 years) age groups, and higher Se levels seen in the middle-aged groups (41-50, 51-60 and 6170 years). Plasma Se levels were found to be significantly lower in males over 81 years when compared to males $41-50$ and males $61-70$ years of age $(89.4 \pm 3.6 \mu \mathrm{g} \mathrm{Se} / \mathrm{L}$ for over 80 years versus $103.5 \pm 2.1$ and $105.3 \pm 2.2 \mu \mathrm{g} \mathrm{Se} / \mathrm{L}$ for $41-50$ and $61-70$ years, respectively). No significant differences were detected for Se levels between males and females within the same

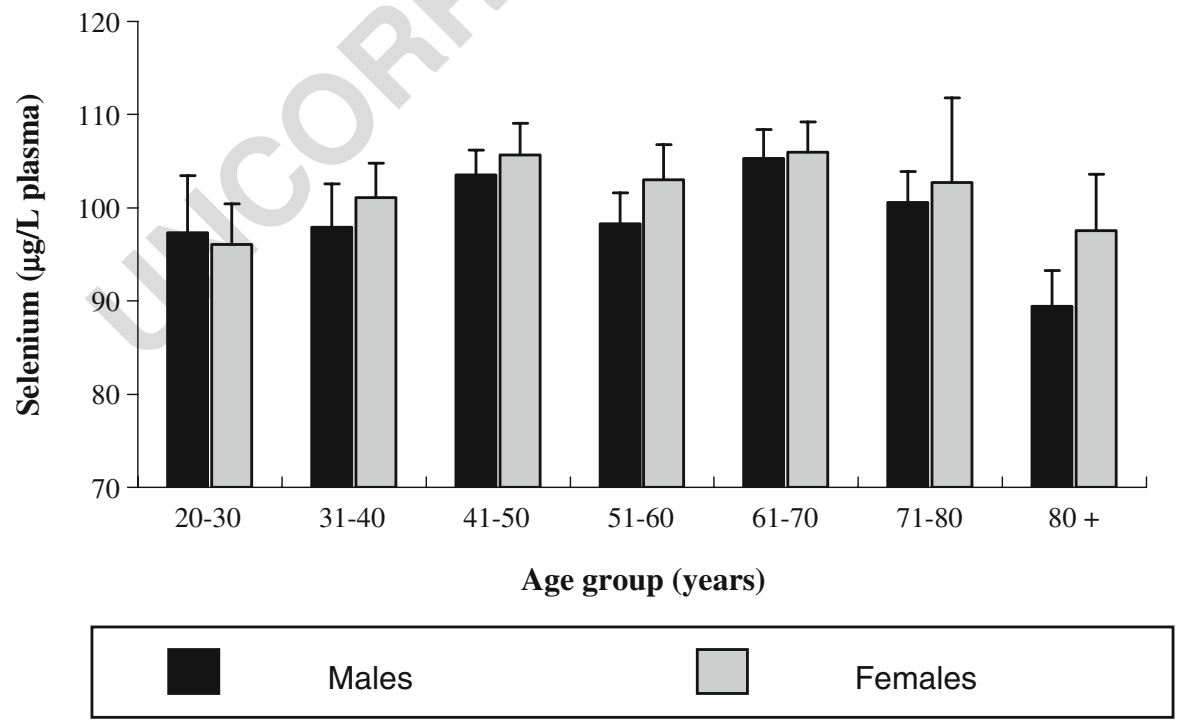

Fig. 2 Effect of age and sex on plasma Se levels ( $\mu$ g Se per L of plasma) in a healthy cohort of the Australian population. Values represent mean \pm SEM. Dotted line indicates plasma Se level required for maximal GSH-Px activity. ${ }^{*} p<0.05$ compared to male $41-50$ years and male $61-70$ years 
Selenium Status of the Australian Population

age groups. GSH-Px activity reflected the trends observed for Se status, with the lowest GSHPx activities observed in the youngest and oldest age groups (Fig. 3). For the males, GSH-Px activity in the 20-30 years and over 81 years of age groups were found to be significantly lower than the 61-70-year age group $(121.5 \pm 5.3 \mathrm{U} / \mathrm{L}$ for the $61-70$ age group versus $98.9 \pm$ 7.1 and $94.2 \pm 7.0 \mathrm{U} / \mathrm{L}$ for the $20-30$ and over 81 years, respectively). As per the Se analysis, there were no significant differences detected between males and females within the same age groups.

\section{Se Status of CVD Patients}

Blood plasma samples from patients presenting for cardiac surgery were analysed for Se levels and GSH-Px activity. The patients presenting for cardiac surgery had a mean age of 66.5 years and $68.9 \%$ were male (Table 1). Of these patients, $12 \%$ were current smokers, whilst $13.5 \%$ had smoked in the past. The patients were presenting for either coronary artery bypass graft (CABG) surgery $(79.3 \%)$ and/or valve repair or replacement surgery $(20.7 \%)$.

The mean plasma Se concentration for the CVD patients was found to be $99.6 \pm 1.7 \mu \mathrm{g} \mathrm{Se} / \mathrm{L}$, which was very similar to the levels observed in the healthy Australians cohort (Table 2). This was also the case for plasma GSH-Px activity, with a mean value of $107.9 \pm 2.2 \mathrm{U} / \mathrm{L}$ for cardiac surgery patients compared to $108.8 \pm 2.0 \mathrm{U} / \mathrm{L}$ for the healthy cohort. However, when the data for cardiac surgery patients and healthy subjects were broken down into age groups, an interesting trend was observed (Figs. 4 and 5). Between the ages of 40-80 years, there was no difference in Se status with CVD, however over the age of 81 years, CVD was associated with a trend for a further decline in both plasma Se $(93.5 \pm 3.6 \mu \mathrm{g} \mathrm{Se} / \mathrm{L}$ for healthy versus $88.2 \pm 5.3 \mu \mathrm{g} \mathrm{Se} / \mathrm{L}$ for CVD, $p=0.21)$ and more so for plasma GSH-Px activity $(98.3 \pm 3.9 \mathrm{U} / \mathrm{L}$ for healthy versus $87.0 \pm 6.5 \mathrm{U} / \mathrm{L}$ for $\mathrm{CVD}, p=0.06$ ). Notably, healthy individuals in this age group also exhibited a sub-optimal Se status.

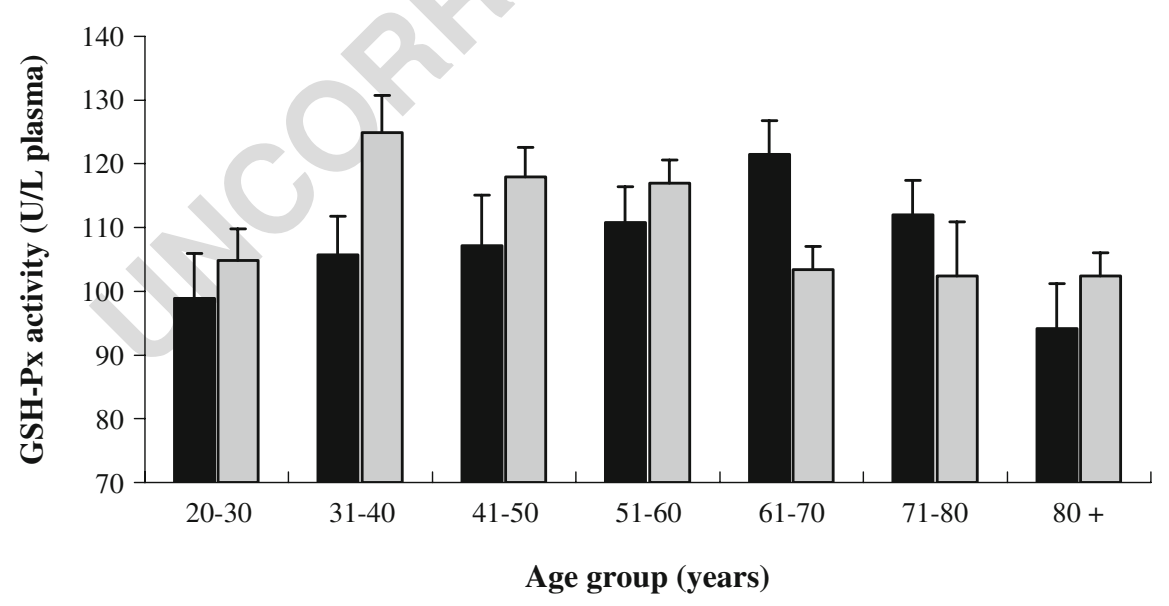

Males

Females

Fig. 3 Effect of age and sex on plasma GSH-Px activity (Units (moles/min) per L of plasma) in a healthy cohort of the Australian population. Values represent mean \pm SEM. ${ }^{*} p<0.05$ compared to male $61-70$ years; $* * p<0.05$ compared to female $31-40$ years 
Lymbury et al.

Table 1 Characteristics of the Cardiac Surgery Patients

$C A B G$ Coronary artery bypass graft surgery, Valve cardiac valve repair or replacement surgery

\begin{tabular}{ll}
\hline & Patient characteristics \\
\hline Mean age (years) & $66.5 \pm 2.4$ \\
Male (\%) & 68.9 \\
Smoking status (\%) & \\
Current & 12 \\
Past & 50 \\
Procedure (\%) & \\
CABG \pm valve & 79.3 \\
Valve only & 20.7 \\
\end{tabular}

\section{Discussion}

Data on dietary Se intake and subsequent plasma Se levels in the Australian population are limited. This cross-sectional study provides a much-needed indication of the Se status of the Australian population, looking at the effects of age, sex and CVD state. However, as discussed below, it also highlights the need for more extensive testing, especially in a country as large and diverse as Australia.

For the healthy Australian population analysed in this study, the mean plasma Se concentration was found to be $100.2 \mu \mathrm{g} / \mathrm{L}$ and the mean GSH-Px activity was found to be 108.8 U/L (Table 3). It has previously been determined that a plasma Se concentration of $100 \mu \mathrm{g} \mathrm{Se} / \mathrm{L}$ or greater is required for optimal plasma GSH-Px activity [8, 11, 14]. The values from the current study revealed that even though the mean value for plasma Se reached the level required for optimal GSH-Px activity, nearly half (47.1\%) of the healthy Australians tested had plasma Se levels below the optimal level. While plasma GSH-Px activity plateaus at a concentration of $100 \mu \mathrm{g} \mathrm{Se} / \mathrm{L}$ plasma, the activity of other selenoproteins, such as platelet GSH-Px [12] and selenoprotein P [15], have been shown to increase further with increasing levels of plasma Se, although this is dependent on the form of Se ingested. Furthermore, there is mounting evidence that higher than optimum intakes of Se may confer a protective effect against certain cancers [16, 17] and CVDs [13]. It has consequently been suggested that the plasma concentration of Se currently deemed as optimum may be an underestimate and that populations may benefit from higher intakes $[8,13,18]$.

Table 2 Mean Plasma Se Concentration ( $\mu$ g per L plasma) and GSH-Px Activity (Units (moles/min) per L Plasma) from Healthy Subjects and Patients Presenting for Cardiac Surgery

\begin{tabular}{lll}
\hline & Healthy & Cardiac surgery \\
\hline $\begin{array}{l}\text { Sample number } \\
\text { Age (years) }\end{array}$ & 100 & 110 \\
$\begin{array}{l}\text { Plasma selenium }(\mu \mathrm{g} / \mathrm{L}) \\
\text { Mean } \pm \text { SD }\end{array}$ & $62.4 \pm 1.4$ & $66.5 \pm 2.4$ \\
$\begin{array}{l}\text { Range } \\
\text { Number of samples less than optimal concentration }\end{array}$ & $101.2 \pm 1.5$ & $99.6 \pm 1.7$ \\
Plasma GSH-Px (Units/L) & 63.0 to 173.0 & 55.5 to 164.7 \\
$\quad$ Mean \pm SD & 44 (44) & $64(57)$ \\
Range & $108.8 \pm 2.0$ & $107.9 \pm 2.2$ \\
\hline
\end{tabular}

${ }^{\text {a }}$ Optimal plasma Se level $=100 \mu \mathrm{g}$ Se per L plasma 
Selenium Status of the Australian Population

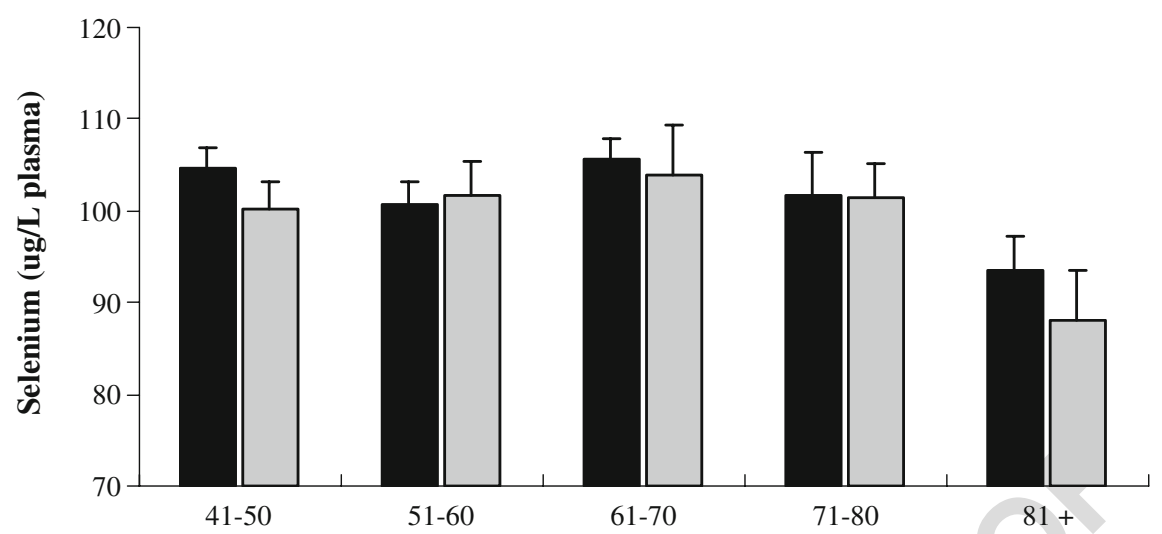

Age group (years)

Fig. 4 Comparison of plasma Se ( $\mu$ g per L plasma) in healthy controls and CVD patients. Values represent mean \pm SEM

Evaluation of the effect of age on Se status in the representative cohort of healthy Australians revealed that the youngest (20-30 years) and oldest ( 81 years and over) age groups were most at risk of a low Se status, with mean plasma Se values falling below the optimal Se level (Figs. 2 and 3). An age-related decline in Se status has previously been reported [19-21] and this was not related to a decline in nutritional intake [20]. Furthermore, recent results of a 9-year longitudinal study of a healthy elderly French population with no evidence of poor nutrition

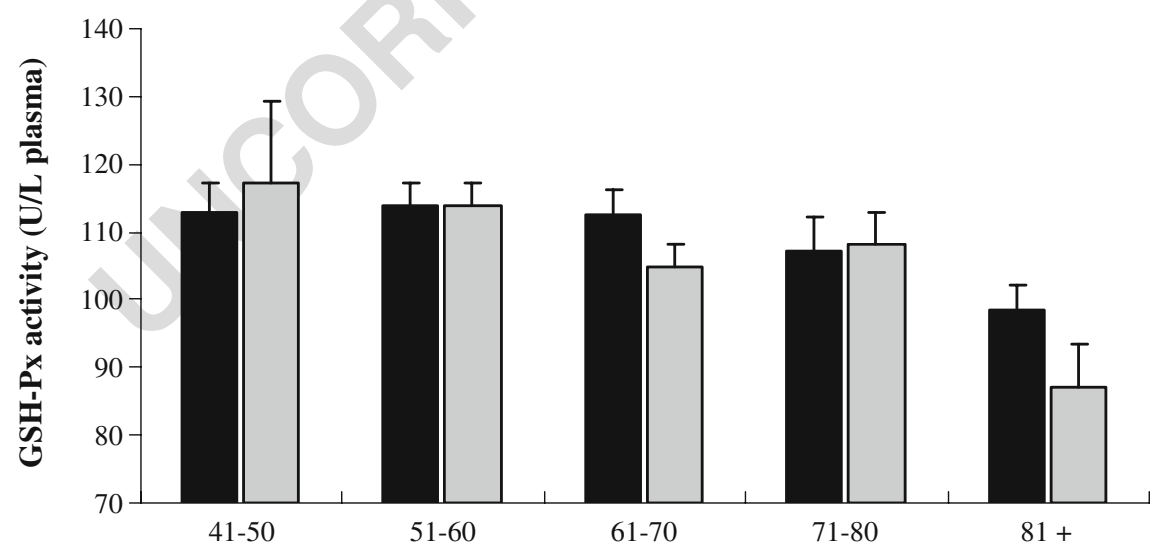

Age group (years)

Fig. 5 Comparison of plasma GSH-Px activity (Units (moles/min) per L plasma) in healthy controls and CVD patients. Values represent mean \pm SEM 
Table 3 Mean Plasma Se Concentration ( $\mu$ g per L plasma) and GSH-Px Activity (Units (moles/min) per L plasma) from Healthy Australian Subjects

\begin{tabular}{llll}
\hline & Male & Female & Combined \\
\hline Sample number & 70 & 70 & 140 \\
Plasma selenium $(\mu \mathrm{g} / \mathrm{L})$ & & & \\
Mean \pm SEM & $98.8 \pm 1.6$ & $101.6 \pm 1.9$ & $100.2 \pm 1.3$ \\
Range & 69.9 to 132.8 & 63.0 to 173.0 & 63.0 to 173.0 \\
Number of samples less than optimal concentration ${ }^{\mathrm{a}}(n(\%))$ & $36(51.4)$ & $30(42.9)$ & $66(47.1)$ \\
Plasma GSH-Px (Units/L) & & & $108.8 \pm 1.7$ \\
Mean \pm SEM & $107.2 \pm 2.9$ & $110.4 \pm 2.1$ & 108.3 to 155.9 \\
Range & 71.5 to 153.6 & 59.3 to 155.9 & 59.3 \\
\hline
\end{tabular}

Values shown are mean \pm SEM

${ }^{\text {a }}$ Optimal plasma Se level $=100 \mu \mathrm{g}$ Se per L plasma

revealed that low baseline plasma Se was significantly associated with higher mortality [4]. Although nutritional indices were not evaluated in the current study, it can be established that in Australia, a well-nourished and developed country, ageing was associated with a decline in Se levels and GSH-Px activity in healthy individuals. Further studies are required to assess the nutritional intake of aging Australians and how this may influence selenium status. There were no significant differences detected for plasma Se or GSH-Px between males and females, however there was a trend for males to have lower levels of Se, especially in the 81 years and over age group. It is plausible that lowered Se levels in males may be associated with the higher morbidity and mortality generally reported in elderly males [22].

Previous reports have suggested that smoking lowers selenium status and GPx activity. In this study all our normal population was non-smokers so we can discount the effect that smoking may have had and the downward trend seen in the older population was not influenced by nicotine intake. However, in any population with cardiovascular disease a high percentage of smokers could be expected, in this study $50 \%$ of patients had previously smoked and $12 \%$ were smoking when the samples were collected. We have analysed the data after excluding the smokers and have seen a similar age-related trend in the cohort with cardiovascular disease (data not shown).

The presence of CVD did not affect Se status in patients aged 41-80 years (Figs. 4 and 5). However, patients aged 81 years and over presenting with CVD demonstrated lower plasma Se and GSH-Px activity compared to healthy subjects of the same age, although this was not found to be significant. While there is evidence for an aetiological role of Se in Keshan Disease and other cardiomyopathies, the results of several epidemiological studies investigating Se and CVD remain inconclusive. Studies conducted in Finland have reported an association between low serum Se levels and the progression of atherosclerosis as well as an increased risk of myocardial infarction (MI) and death from ischemic heart disease (IHD) [23]. A Danish study also demonstrated an increased risk of IHD in subjects with a serum Se level below $79 \mu \mathrm{g} / \mathrm{L}$ [24]. The EURAMIC study, a multi-centre study based in Europe and Israel, reported a significant association between Se and IHD, but only in the centre with the lowest Se status [25]. Conversely, no significant associations were found in studies conducted in Helsinki [26], Norway [27], the Netherlands [28] and the United States [29]. These inconsistent findings are most likely attributable to a biological threshold effect of Se, with significant risks observed only in seleno-deficient populations. The findings from the current Australian study are consistent with this. Age groups that were seleno-replete or of marginal 
Selenium Status of the Australian Population

individuals over the age of 81 showed a lowered Se status, and this was further exacerbated in CVD patients. Furthermore, studies have shown that mortality following cardiac surgery and $\mathrm{MI}$ is three times higher in patients older than 70 years compared to younger age groups $[30,31]$. It should be noted that low Se status is only one factor and any associations do not conclusively indicate causality. However, considering that no associations are observed in populations with high Se status, whilst significant associations are observed in populations with low to moderate Se status, it is of significant public health interest to ensure an adequate Se status for all Australians, in particular the elderly, as highlighted in this study.

In comparison to other countries, Australian Se values are considered to be moderate, being substantially higher than New Zealand (53-65 $\mu \mathrm{g}$ Se/L) [11, 32] and the United Kingdom (63 $\mu \mathrm{g} \mathrm{Se} / \mathrm{L})$ [14], whilst lower than the United States (80-167 $\mu \mathrm{g} \mathrm{Se} / \mathrm{L})$ [8]. The estimated world mean is $78 \mu \mathrm{g} \mathrm{Se} / \mathrm{L}$, and it has been estimated by Combs [8] that the number of people worldwide that are at risk of Se deficiency is in the range of 500 million to one billion [8], and this was not taking into account marginal Se deficiency. Over the past 10 to 20 years, there has been a concerning trend for declining Se levels in soil and food systems. This has been reported internationally [14] and also here in Australia [33] and is most likely attributable to more intensive cropping, use of sulphur-containing fertilisers (sulphur competes with Se) and the burning of fossil fuels leading to acid rain and soil acidification [33].

The results of the current study demonstrate that nearly half of the healthy Australian cohort tested have marginal Se levels. This study also highlights the importance of an adequate Se status in the ageing Australian population, especially in terms of maintaining cardiovascular health. Given these findings, as well as the increasing evidence of an aetiological role for Se in several disease states, further investigations into the status of Se in the Australian population is warranted.

\section{References}

1. Navarro-Alarcon M, de la Serrana HL, Perez-Valero V, Lopez-Martinez C (1998) Serum selenium levels as indicators of body status in cancer patients and their relationship with other nutritional and biochemical markers. Sci Total Environ 212:195-202

2. Navarro_Alarcon M, Lopez_Martinez MC (2000) Essentiality of selenium in the human body: relationship with different diseases. Sci Total Environ 249:347-371

3. Wei WQ, Abnet CC, Qiao YL, Dawsey SM, Dong ZW, Sun XD, Fan JH, Gunter EW, Taylor PR, Mark SD (2004) Prospective study of serum selenium concentrations and esophageal and gastric cardia cancer, heart disease, stroke, and total death. Am J Clin Nutr 79:80-85

4. Akbaraly NT, Arnaud J, Hininger-Favier I, Gourlet V, Roussel AM, Berr C (2005) Selenium and mortality in the elderly: results from the EVA study. Clin Chem 51:2117-2123

5. Arnaud J, Akbaraly NT, Hininger I, Roussel AM, Berr C (2007) Factors associated with longitudinal plasma selenium decline in the elderly: the EVA study. J Nutr Biochem 18:482-487

6. Blankenberg S, Rupprecht HJ, Bickel C, Torzewski M, Hafner G, Tiret L, Smieja M, Cambien F, Meyer J, Lackner KJ (2003) Glutathione peroxidase 1 activity and cardiovascular events in patients with coronary artery disease. N Engl J Med 349:1605-1613

7. Flores-Mateo G, Navas-Acien A, Pastor-Barriuso R, Guallar E (2006) Selenium and coronary heart disease: a meta-analysis. Am J Clin Nutr 84:762-773

8. Combs GF Jr (2001) Selenium in global food systems. Br J Nutr 85:517-547

9. Judson GJ, Reuter DJ (1999) Selenium. In: Peverill KI, Sparrow LA, Reuter DJ (eds) Soil analysis: an interpretation manual. CSIRO Publishing, Melbourne, Australia, pp 325-329

10. Reilly C (1996) Selenium in food and health, 2nd edn. Blackie Academic and Professional, London, UK

11. Thomson CD, Robinson MF, Butler JA, Whanger PD (1993) Long-term supplementation with selenate and selenomethionine: selenium and glutathione peroxidase (EC 1.11.1.9) in blood components of New Zealand women. Br J Nutr 69:577-588

12. Neve J (1995) Human selenium supplementation as assessed by changes in blood selenium concentration and glutathione peroxidase activity. J Trace Elem Med Biol 9:65-73

13. Rayman MP (2000) The importance of selenium to human health. Lancet 356:233-241 